\title{
De novo developed microsatellite markers in gill parasites of the genus Dactylogyrus (Monogenea): revealing the phylogeographic pattern of population structure in the generalist parasite Dactylogyrus vistulae
}

\author{
Michal Benovics ${ }^{1}$, Lenka Gettová ${ }^{1}$, and Andrea Simková ${ }^{1}$ \\ ${ }^{1}$ Masaryk University
}

July 21,2021

\begin{abstract}
Approaches using microsatellite markers are considered the gold standard for modern population-genetic studies. However, though they have found application in research into various platyhelminth taxa, they remained substantially underutilized in the study of monogeneans. In the present study, a newly-developed set of 24 microsatellite markers was used to investigate the genetic diversity of the generalist monogenean species D. vistulae. The analyzed parasite specimens were collected from 13 cyprinoid species from 11 sites in the Apennine and Balkan peninsulas. A total of 159 specimens were genotyped at each of the loci and the number of alleles per locus ranged from 2 to 16, with a mean number of 6.958 alleles per locus. Exceptionally high genetic diversity was observed among D. vistulae individuals in the southern Balkans, suggesting that this region might represent the center of diversification of the genus in Europe, from where Dactylogyrus parasites expanded into the north. The initial clustering analysis divided all investigated specimens into three major clusters; however, the results of the subsequent analyses revealed the existence of various subpopulations, suggesting that the population structure of D. vistulae is associated with the diversification of their cyprinoid hosts. In addition, partition of the parasite population was observed in regions of the sympatric occurrence of two host species, indicating that these hosts may represent a barrier to gene flow, even for generalist parasite species.
\end{abstract}

De novo developed microsatellite markers in gill parasites of the genus Dactylogyrus (Monogenea): revealing the phylogeographic pattern of population structure in the generalist parasite Dactylogyrus vistulae

Running title: Microsatellite markers for monogeneans

Michal Benovics ${ }^{1,2 *}$, Lenka Gettová ${ }^{1}$, Andrea Šimková ${ }^{1}$

${ }^{1}$ Department of Botany and Zoology, Faculty of Science, Masaryk University, Kotlářská 2, 611 37, Brno, Czech Republic

${ }^{2}$ Department of Zoology, Faculty of Sciences, Comenius University in Bratislava, Mlynská dolina, Ilkovičova 6, 84215 Bratislava, Slovakia

*corresponding author's e-mail: benovics@mail.muni.cz

\section{Abstract}

Approaches using microsatellite markers are considered the gold standard for modern population-genetic studies. However, though they have found application in research into various platyhelminth taxa, they 
remained substantially underutilized in the study of monogeneans. In the present study, a newly-developed set of 24 microsatellite markers was used to investigate the genetic diversity of the generalist monogenean species $D$. vistulae. The analyzed parasite specimens were collected from 13 cyprinoid species from 11 sites in the Apennine and Balkan peninsulas. A total of 159 specimens were genotyped at each of the loci and the number of alleles per locus ranged from 2 to 16, with a mean number of 6.958 alleles per locus. Exceptionally high genetic diversity was observed among D. vistulae individuals in the southern Balkans, suggesting that this region might represent the center of diversification of the genus in Europe, from where Dactylogyrusparasites expanded into the north. The initial clustering analysis divided all investigated specimens into three major clusters; however, the results of the subsequent analyses revealed the existence of various subpopulations, suggesting that the population structure of $D$. vistulae is associated with the diversification of their cyprinoid hosts. In addition, partition of the parasite population was observed in regions of the sympatric occurrence of two host species, indicating that these hosts may represent a barrier to gene flow, even for generalist parasite species.

\section{Keywords}

Population genetics, Polymorphic markers, Cyprinoidei, Historical dispersion, Host-specific parasites

\section{Introduction}

The monogenean genus Dactylogyrus Diesing, 1850 is the most speciose taxon among Platyhelminthes. According to the checklist compiled by Gibson, Timofeeva and Gerasev (1996), this genus includes more than 900 nominal species, of which the majority are gill parasites. However, this number is strongly underestimated considering the diversity of their common hosts - freshwater fish of the suborder Cyprinoidei (classification following the recent studies of Tan and Armbruster (2018), and Schönhuth, Vukić, Šanda, Yang and Mayden (2018)). The majority of species belonging to Dactylogyrus are gill parasites. The biogeographical distribution of Dactylogyrusconcurs with the distribution of their cyprinoid fish hosts, as their occurrence in native cyprinoids was documented in Africa, Asia, North America, and Europe (e.g., Hoffman, 1999; Pugachev, Gerasev, Gussev, Ergens \& Khotenowsky, 2009; Řehulková, Seifertová, Přikrylová \& Francová, 2018). Each cyprinoid species potentially serves as a host for at least one Dactylogyrus species, and different lineages ofDactylogyrus parasitize divergent host lineages (e.g., Šimková, Morand, Jobet, Gelnar \& Verneau, 2004; Šimková, Benovics, Rahmouni \& Vukić, 2017; Benovics, Desdevises, Vukić, Šanda \& Šimková, 2018; Benovics et al., 2020a; Řehulková, Benovics, Šimková, 2020). In respect to their hosts, Dactylogyrus parasites exhibit different levels of specificity. They range from strict specialists which parasitize a single host species, through intermediate specialists parasitizing congeneric hosts and phylogenetic specialists parasitizing closely related hosts, to true generalists parasitizing phylogenetically distant host species i.e. hosts belonging to different families (Šimková, Verneau, Gelnar \& Morand, 2006; Kuchta et al., 2020).

Dactylogyrus vistulae Prost, 1967 is the most striking example of generalist Dactylogyrus, evidenced from 24 cyprinoid species of six genera in the Balkans and Central Europe (Benovics et al., 2018). This species was also reported from other European regions (e.g., Nordic countries; Koskivaara and Valtonen (1992), Southwest Europe; Seifertová, Vyskočilová, Morand and Šimková (2008), Apennine Peninsula; Benovics, Francová, Volta, Dlapka and Šimková, 2021) and the Middle East (e.g., Aydogdu, Altunel \& Yildirimhan, 2001; Mhaisen \& Abdul-Ameer, 2019). In comparison to the majority of Dactylogyrus species, which generally parasitize only the hosts from phylogenetically related lineages, the host range of $D$. vistulae encompasses a variety of phylogenetically divergent cyprinoid genera i.e., genera belonging to the families Cyprinidae and Leuciscidae (Moravec, 2001; Benovics et al., 2018; 2021), between which D. vistulae presumably often host-switch (suggested by Benovics, Desdevises, Śanda, Vukić and Šimková (2020b). Benovics et al. (2018) investigated the molecular diversity of $D$. vistulae in the Balkans using partial 18S, ITS1, and partial 28S rDNA. They found 13 genetic variants in the Balkans and two genetic variants in the Czech Republic (representing Central European samples) representing different populations, i.e. parasite populations associated with different host species or parasite populations associated with geographically distant populations of the same host species. Moreover, Benovics et al. (2018) showed a strong correlation between the genetic distances and geographical distances of $D$. vistulae populations, suggesting that geographical separation played a more critical role in 
the divergence of $D$. vistulae populations than their hosts' phylogenetic relationships. Besides its remarkably wide host range and distribution range, $D$. vistulae is also easily distinguishable from other congeners due to its relatively large body size, and the size and simplicity of taxonomically important features (Pugachev et al., 2009), which promote $D$. vistulae as the optimal candidate species for population-genetic studies in dactylogyrid monogeneans.

Over the last few decades, approaches using microsatellite markers (together with mitochondrial DNA) have become the gold standard for most population-genetic studies. These highly polymorphic short tandem repeats are, due to their unique characteristics (e.g., high allelic variance, codominance, and Mendelian inheritance), usually applied to infer gene flow rate, hybridization, or mating pattern on the intra- and interpopulation levels. Studies investigating interpopulation variability in monogeneans are often conducted using either morphometrics (e.g., Mariniello, Ortis, D'Amelio \& Petrarca, 2004; Vignon \& Sasal, 2010; Rodríguez-González, Míguel-Lozano, Llopis-Belenguer \& Balbuena, 2015; Khang, Soo, Tan \& Lim, 2016; Kmentová et al., 2016; Rahmouni, Van Steenberge, Vanhove \& Šimková, 2020), mitochondrial markers (e.g., Huyse, Oeyen, Larmuseau \& Volckaert; 2017; Antonio Baeza, Sepúlveda \& Teresa González, 2019; Pettersen, Junge, Østbye, Mo \& Vøllestad, 2021), or the combination of both methods (e.g., Huyse \& Volckaert, 2002; Kmentová et al., 2020a;b). However, until now, no genetic markers useful for revealing population structure in monogeneans have been identified for studies on dactylogyrids. Studying the population structure of parasites in association with their hosts - in our study, Dactylogyrus vistulae associated with cyprinoids mainly in Europe - may either reveal the geographical isolation between different cyprinoid species (or populations of the same cyprinoid species) or, alternatively, indicate secondary contacts between the hosts. Microsatellite markers are still underutilized in this platyhelminth group, and, to date, only one study designing primers for polymorphic repeats in gyrodactylids has been conducted, this by Faria, Lazarus, Oosterhout, Harris and Cable (2011). Therefore, the aim of this study was (1) to design a set of microsatellite primers for the widely distributed generalist monogenean species $D$. vistulae using next generation sequencing, and (2) to test their functionality with respect to revealing interpopulation genetic variability in order to investigate the geographical structure of the populations of this species.

\section{Material and Methods}

\section{Material}

To cover geographically distant regions within the distribution range of $D$. vistulae, parasites were collected from cyprinoid hosts at 29 sites across the Balkan and Apennine Peninsulas. However, sites at which fewer than 5 D. vistulae individuals were collected were discarded from the final dataset; therefore, individuals from 11 sites in the Balkans and one site in the Apennine Peninsula were included in this study. All included D, vistulae individuals were collected from 13 host species - six species of Squalius, four species of Telestes , two species of Phoxinellus, and Chondrostoma phoxinus (Table 1).

\section{Preparation of samples for NGS and identification of polymorphic tandem repeats}

The whole genomic sequencing was performed from the pooled sample containing $D$. vistulae individuals collected from Squalius cephalus from the Dyje River (Czech Republic). Due to the focus of the paper on southern European regions with an intertwined geographic history, data on the $D$. vistulae population from the Czech Republic were omitted from further analyses. Genomic DNA was isolated using DNEasy Blood \& Tissue Kit (Qiagen, Hilden, DE). Subsequently, library preparation using KAPA LTP Preparation Kit (Kapa Biosystems, Century City, SA) and sequencing on an Illumina Miseq platform (Illumina) using Miseq Reagent Kit v2 (Illumina) were provided by CEITEC (Masaryk University, Brno, CZ). The QDD (Meglécz et al., 2010) program was used to select 104 sequences with target microsatellites according to the following conditions: (i) the target microsatellites had at least seven repetitions, (ii) the length of the PCR product was between 100 and $300 \mathrm{bp}$, (iii) flanking regions did not contain a mononucleotide stretch of more than four bases, or any di-hexa base pair motifs of more than 100 two repetitions, (iv) the annealing temperatures of primer pairs were optimized to fall between $57{ }^{\circ} \mathrm{C}$ and $63{ }^{\circ} \mathrm{C}$, (v) microsatellites were not compound or interrupted, and (vi) selected sequences were not of fish origin based on BLAST results in the GenBank 
database.

PCR amplification, sequencing, and selection of microsatellite loci

Selected microsatellite primers were first tested separately in PCR reactions performed in a final volume of $10 \mu \mathrm{l}$, comprising $0.5 \mu \mathrm{l}$ of template DNA, $0.2 \mathrm{mM}$ dNTP, $1 \times \mathrm{PCR}$ buffer, $2.0 \mathrm{mM} \mathrm{MgCl}_{2}, 0.5 \mathrm{U}$ Taq DNA polymerase (Invitrogen, Waltham, MA), and $0.2 \mu \mathrm{M}$ of each of the forward and reverse primers. The conditions of the PCR reaction were as follows: initial denaturation at $94{ }^{\circ} \mathrm{C}(2 \mathrm{~min}), 35$ cycles of denaturation at $94{ }^{\circ} \mathrm{C}(60 \mathrm{~s})$, annealing at $51-63{ }^{\circ} \mathrm{C}(30 \mathrm{~s})$, an extension at $72{ }^{\circ} \mathrm{C}(2 \mathrm{~min})$, and a final extension at $72{ }^{\circ} \mathrm{C}(10$ $\min )$. The PCR products were visualized by $2 \%$ agarose gel electrophoresis. Microsatellites that produced unreliable bands, or positive bands for the control sample represented by DNA of the fish host, were further excluded. The remaining microsatellites were tested under the same PCR conditions as described above, with the forward primer labeled by fluorescent dye (i.e. PET, NED, VIC or FAM) and analyzed on an ABI PRISM 3130 Genetic Analyzer (Applied Biosystems, Foster City, CA) using 500 LIZ Size Standard (Applied Biosystems, Foster City, CA). Genotypes were scored using GeneMapper Software version 4.0 (Applied Biosystems, Foster City, CA). Finally, 24 polymorphic microsatellites were pooled into the five multiplex PCR sets. PCR reactions were carried out in a total volume of $10 \mu \mathrm{l}$ using the Qiagen Multiplex PCR Kit (Qiagen, Hilden, DE) with 35 cycles and an annealing temperature of $52^{\circ} \mathrm{C}$ (Table 2).

\section{Genetic analyses}

In total, 159 D. vistulae individuals were identified initially using morphological characters (shape and sizes of anchors, marginal hooks, connective bar, and sclerotized parts of male copulatory organ and vagina). Their species identities were evaluated by amplification of the partial genes coding $18 \mathrm{~S}$ rDNA and $28 \mathrm{~S}$ rDNA (following Benovics et al. (2018)), and subsequent comparison to the sequences deposited in the GenBank. For microsatellite markers, the number of alleles (NA), the number of effective alleles (Ne), Shannon's diversity index, the observed $\left(\mathrm{H}_{\mathrm{O}}\right)$ and expected $\left(\mathrm{H}_{\mathrm{E}}\right)$ heterozygosity, the fixation index (i.e. $\mathrm{F}$, proportion of homozygotes), $\mathrm{F}_{\mathrm{ST}}$ (i.e., genetic variance in allele frequencies), and the Nei-distances (i.e., the number of eigen differences) were computed using the program GenAlEx v 6.5 (Peakall \& Smouse, 2006; 2012). In order to test whether microsatellite markers are suitable for the identification of population structure in $D$. vistulaemonogeneans, two methods were used: Bayesian clustering analysis, which was conducted in Structure v 2.3.4 (Pritchard, Stephens \& Donnelly, 2000; Falush, Stephens \& Pritchard, 2007) and multivariate Principal Coordinate Analysis (PCoA) implemented in GenAlEx v 6.5. An admixture model with expected uncorrelated allele frequencies was applied for the clustering analysis in Structure v 2.3.4. The expected number of clusters $(\mathrm{K})$ was tested within the range of 1 to 15 . Basically $\mathrm{K}$ range was estimated, on the basis that each parasite population was associated with an individual collection site (a total of 12 collection sites). However, to test whether further fragmentation occurs between sympatric hosts, or even within a single host population, the number of $\mathrm{K}$ was increased to 15 . A series of ten independent runs with $10^{7}$ Markov-chain Monte Carlo $\left(\mathrm{MC}^{2}\right)$ iterations, the initial $10^{6}$ iterations discarded as a burn-in, were conducted for each tested K. The most optimal K (i.e., with the highest likelihood) was selected using the Structure Harvester (Evanno, Regnaud \& Gould, 2005; Earl \& vonHoldt, 2012) and plot graphs were visualized using CLUMPAK (Kopelman, Mayzel, Jakobsson, Rosenberg \& Mayrose, 2015).

\section{Results}

\section{Allelic diversity in microsatellite loci}

From all candidate loci, which were selected according to the results of PCR amplification and sequencing tests, 24 were detected to be polymorphic and were chosen for further population analyses. The genetic diversity of each locus per collection site, including range of alleles, total number of alleles per locus $\left(\mathrm{TN}_{\mathrm{A}}\right)$, number of alleles per population $\left(\mathrm{N}_{\mathrm{A}}\right)$, and observed and expected allele heterozygosity per population $\left(\mathrm{H}_{\mathrm{O}}\right.$, $\mathrm{H}_{\mathrm{E}}$ ) are presented in Table 3. The number of alleles per locus ranged from 2 (locus 75) to 16 (locus 73 ) with a mean number of 6.958 alleles per locus. In respect to specific geographic sites (representing different host species), the highest level of genetic polymorphism was recorded in $D$. vistulae individuals parasitizing north-western Greek Squalius prespensis (site G1). Individuals from this site exhibited the highest mean 
heterozygosity across all loci $\left(\mathrm{H}_{\mathrm{O}}=0.453\right)$, the highest number of alleles per individual $(\mathrm{Na}=3.917)$, and the highest mean number of effective alleles $(\mathrm{Ne}=2.662)$. The descriptive statistics indicating mean values for basic population parameters for all loci in respect to the collection sites are reported in Table 4. In general, each population exhibited low genetic variability and encompassed a high proportion of homozygotic individuals, i.e. the majority of loci were monomorphic in almost all populations and only interpopulation allele lengths were observed. Homozygotic individuals were present at five of the 12 collection sites. The only locus which did not amplify across all individuals from a single collection site was locus 96 in G1. Neidistances and $\mathrm{F}_{\mathrm{ST}}$ were computed for each population pair and are reported in Table 5. Nei-distances ranged from 0.022 to 1.731 and the greatest distances were found between the G2 population and each of the C4 and C5 populations. Pairwise $\mathrm{F}_{\mathrm{ST}}$ ranged from 0.103 to 0.959 and the greatest distances were observed between population $\mathrm{C} 4$ and the following populations: B1, B2, C1, C2 and C3. A similar pattern was revealed for the C5 population.

\section{Population structure}

The mean estimated likelihood of ten runs from all $\mathrm{K}$ tested by Bayesian clustering analyses peaked at $\mathrm{K}=$ 8 , revealing a structured dataset encompassing 8 population clusters (Figure 2A). However, the estimated $\Delta \mathrm{K}$ peaked at $\mathrm{K}=3$ (Figure $2 \mathrm{~B}$ ), suggesting that major genetic structuration is observed for 3 estimated populations. Bar plots visualizing the proportion of the genome of each individual belonging to a particular cluster resulting from the Structure are shown for $\mathrm{K}=3, \mathrm{~K}=5$, and $\mathrm{K}=8$ in Figure 3. The initial lower estimated $\mathrm{K}$ divided all investigated $D$. vistulae individuals into three major clusters $(\mathrm{K}=3)$. The first one encompassed individuals collected from sites in Bosnia and Herzegovina (B1-B4), and southern Croatia (C1C3). The second cluster encompassed all individuals from Greek sites G1 and G2. Finally, the third cluster encompassed individuals collected from sites in central Croatia (C4 and C5), and individuals parasitizing T. muticellus in Italy (termed as I1b). The D. vistulae individuals parasitizing $S$. squalus from the same site in Italy (I1a) were genetically ambiguously associated with all three clusters. Gradually increasing the number of estimated K first separated Greek D. vistulae individuals into two clusters, each associated with the respective collection site (G1 and G2), and later separated the Apennine population parasitizing $T$. muticellus $(\mathrm{K}=5)$. In addition, the existence of three subpopulations was suggested $\operatorname{among} D$. vistulae from sites B1-B4 and C1-C3; however, the results from both clustering analyses clearly document gene flow between these populations representing seven collection sites in Bosnia and Herzegovina, and Croatia $(\mathrm{K}=$ 8 ). At the higher estimated $\mathrm{K}(<7)$, clustering analysis divided the individuals from Italy into two separate subpopulations - each associated with the respective host species. The separation of I1 subpopulations also indicates genetic drift, whereas the observed heterozygosity in population $\mathrm{I} 1 \mathrm{~b}\left(\mathrm{H}_{\mathrm{O}}=0.004\right)$ is significantly lower in comparison to population I1a $\left(\mathrm{H}_{\mathrm{O}}=0.192\right.$, Table 4$)$. The results of PCoA were congruent with the structure revealed by Bayesian clustering analysis (i.e. the separation of populations C4, C5, G1, G2, and I, and the grouping of individuals from sites B1-B4 together with C1-C3, Figure 4a). Moreover, the analysis also visibly separated the subpopulations from site I1 on the x-axis. Subsequent PCoA analysis including only individuals from the latter cluster in segments 2 and 3 supported the partial separation of subpopulations from B4 and C3, as was suggested by Bayesian clustering analysis with higher estimated K (Figure 4b).

\section{Discussion}

The suitability of $D$. vistulae for population-genetic studies of monogenean parasites was previously suggested by Benovics et al. (2018). Prior to this study, there were no molecular markers known to study the population genetics of gill monogeneans. Therefore, in this paper, we present de novo microsatellite markers for dactylogyrids, which we applied in the interpopulation study of generalist Dactylogyrus species exhibiting a wide distributional range (including a wide range of host species).

We used 24 microsatellite markers to investigate the molecular diversity of populations of the generalist parasite $D$. vistulae. We found low allele variance in all studied markers at ten out of the 12 sites in the north-eastern peri-Mediterranean, where $D$. vistulae was collected from 13 host species. High allelic diversity was observed in individuals from the two remaining sites (two parasite populations); Aoos in 
north-west Greece, and the Cerfone Stream (Tiber drainage) in Italy.

The exceptionally high genetic diversity in the Greek population may suggest a relatively more ancestral origin for this population than in the case of other Balkan populations. Assuming that the phylogeographic origin and historical dispersion of parasites are intimately linked with the phylogeography of their hosts, this claim is also supported by the historical dispersion route proposed for cyprinoids via the continental bridge dividing Paratethys and connecting the Balkan and Anatolian landmass (Steininger \& Rögl, 1984; Doadrio, 1990; Perea et al., 2010). In such a case, subsequent dispersion into the Balkan Peninsula occurred from south to north, and, therefore, the molecular diversity of $D$. vistulae is much lower due to the founder's effect. A similar argument might be applied to explain the molecular diversity of $D$. vistulae in the Peloponnese. Even though the observed heterozygosity of Peloponnesian $D$. vistulae populations is higher than that of central Balkan populations, it might simply reflect the more ancestral diversification and subsequent isolation of parasite populations (or their host species) in the Peloponnese peninsula. The other factor that potentially impacted the formation of parasite populations in north-western Greece is the historical formation of the Dessaretes lake system, which occurred in this region and played an essential role in the speciation of cyprinoids in the Balkans. This large connection of water bodies originated in the Pliocene and covered the area of all the present Great Lakes of the Balkan Peninsula; i.e., Ohrid Lake (located on the border of Albania and North Macedonia), Prespa Lake (Albania, Greece, and North Macedonia), Mikri Prespa Lake (Albania and Greece), and Maliq Lake (Albania, evaporated during World War II) (Sušnik, Snoj, Wildon, Mrdak \& Weiss, 2007; Abell et al., 2008; Albrecht \& Wilke, 2008, Schultheiss, Albrecht, Bossneck \& Wilke, 2008; Bordon, Peyron, Lezine, Brewer \& Fouache, 2009; Wagner \& Wilke, 2011). Later, after the closing of the Korca depression and connections between Paratethys and Dessaretes, the water level gradually decreased, promoting allopatric speciation in the freshwater fish fauna and leading to recent rich species diversity in the Great Lakes (Steininger \& Rögl, 1984; Albrecht \& Wilke, 2008). However, past underground hydrological connections between already divided lakes and neighboring drainages (as suggested by Albrecht et al. (2008)) facilitated the mixing of parasite populations between diversified host species and, therefore, gradually increased genetic diversity. At present, S. prespensis is an endemic species of lakes of the Prespa basin (Kottelat \& Freyhof, 2007); therefore, we can assume that this species may share parasite communities genetically related to parasite communities of other endemic cyprinoids in the region of the former Dessaretes. However, thorough investigation of parasite communities, including, in particular, population analyses of widely distributed parasite species, is required to validate such a hypothesis.

In contrast to Greek populations, the high level of polymorphism in Italy can be explained by the syntopy of two co-occurring hosts of $D$. vistulae in the region. In the Torrente Cerfone, $D$. vistulae individuals were collected from two cyprinoid species,S. squalus and T. muticellus. Clustering analyses revealed that each host species harbors a genetically divergent population of D. vistulae (Figure 3). However, the subsequent comparison of genetic variability between $D$. vistulae individuals parasitizing $S$. squalus and $D$. vistulae individuals parasitizing T. muticellus detected a high level of polymorphism only in the former population. A possible explanation for the observed difference in genetic polymorphism might be the putative origin of $D$. vistulae of the Apennine Peninsula. It has been hypothesized that during the Last Glacial Maximum, when the sea level drastically regressed (Pielou, 1979; Bianco, 1990; Waelbroeck et al., 2002), the expansion of the Po basin facilitated contact between freshwater fauna of the currently isolated Italian and Balkan river systems (Waelbroeck et al., 2002; Stefani, Galli, Crosa, Zaccara \& Calamari, 2004). Such a connection would also explain the fact that cyprinoid fauna (e.g., S. squalus ) shows no or very little molecular divergence between species living on both sides of the Adriatic Sea (Buj et al., 2010; Perea et al., 2010; Geiger et al., 2014). During this time, S. squalus (or its ancestor) dispersed together with other cyprinoids and their parasites from the Balkans to Apennine Peninsula. A high level of genetic polymorphism in D. vistulae may suggest that $S$. squalus served as a main host species for the dispersion of this parasite in the Apennine Peninsula, where this parasite later host-switched to endemic species of Telestes. This hypothesis is also supported by the low genetic polymorphism in the D. vistulae population harbored by $T$. muticellus and by the calculated pairwise Nei-distances and $\mathrm{F}_{\mathrm{ST}}$. The population from $S$. squalus is genetically more similar to the central Balkan populations (Nei-distances $<0.509, \mathrm{~F}_{\mathrm{ST}}<0.388$ ) than to the population from T. muticellus (Nei- 
distance $=0.794, \mathrm{~F}_{\mathrm{ST}}=0.447$, Table 4). Despite the fact that the historical dispersion of $D$. vistulae included host switching between species representing phylogenetically close genera, the current hosts of $D$. vistulae in Torrente Cerfone apparently represent a barrier reducing the gene flow between parasite populations, even when host species live in syntopy, and biological requirements and habitats strongly overlap (see Kottelat and Freyhof (2007) for the biology and distribution of host species).

Initial clustering analyses separated all the studied populations into three major clusters, each essentially associated with the different ichthyogeographical regions (sensu Bianco, 1990). The clustering of individuals from the Apennine Peninsula and D. vistulae of the Balkan T. croaticus and T. fontinalis (sites C4 and C5, respectively) follows the range of the Padano-Venetian district, which covers the region from the River Vomano in central Italy to the River Krka in Dalmatia. The range of this district essentially corresponds with the aforementioned Po basin during the glacial period. Moreover, these two Telestes species also represent a distant lineage from remaining congeners in the Balkans, whose ancestor diverged in the middle of the Miocene (Buj et al., 2017), which explains why theD. vistulae populations harbored by T. croaticus and $T$. fontinalis are divergent from other D. vistulaepopulations in the western Balkans. Thus, the second cluster encompassing individuals from four sites in Bosnia and Herzegovina and three southern Croatian sites is in accordance with the Dalmatian district's range, which corresponds with the distribution range of $S$. svallize (Bianco, 1990; Kottelat \& Freyhof, 2007). The Dalmatian ichthyogeographic district almost exclusively encompasses endemic cyprinoid species. The subsequent clustering analyses (Figure 3; K8, Figure 4b) separated individuals of $S$. tenellus (site B4) and T. turskyi (C3) into individual subpopulations; however, it also clearly indicated gene flow between $D$. vistulae populations sampled in Bosnia and Herzegovina, and those from Croatia. Both $S$. tenellus and T. turskyi are highly endemic with a strongly limited distribution range. While $S$. tenellus is primarily endemic to the Cetina River, some populations can also be found in nearby karstic streams; for example, T. turskyi is restricted to the Cikola River (Kottelat \& Freyhof, 2007). In general, almost all endemic cyprinoid species in the Dalmatian district have an extremely limited distribution range, often limited to a single river stream in the karst (Bianco, 1990; Kottelat \& Freyhof, 2007). Therefore, gene flow between populations of freshwater fish and between their parasites as well, should be minimal. Nonetheless, the low genetic variance and the results of clustering analyses suggest the opposite. The partial mixing of parasite populations could result from recent contacts between hosts from geographically "isolated" regions via underground connections proposed by Palandačić, Bravničar, Zupančič, Šanda and Snoj (2015). However, there is no doubt that the observed population structure is associated with the distribution of endemic hosts in the Balkans, and, from the low degree of heterozygosity we can assume that the subpopulations are of relatively recent origin.

Microsatellite markers represent a powerful molecular tool in population-genetic studies of parasites. These polymorphic tandem repeats were previously employed in studies of the population structures of medically important human parasites (e.g., Schistosoma haematobium, Gower et al., (2011)). However, they are still strongly underutilized in studies of parasitic platyhelminths in wildlife, as only a few papers focusing on cestodes (i.e., Luo, Nie, Zhang, Yao \& Wang, 2003; Štefka, Hypša \& Scholz, 2009; Umhang et al., 2018; Bazsalovicsová et al., 2020) or digeneans (i.e., Criscione \& Blouin, 2006; Criscione, Cooper \& Blouin, 2006; Louhi, Karvonen, Rellstab \& Jokela, 2010; Criscione, Vilas, Paniagua \& Blouin, 2011; Valdivia, Criscione, Cárdenas, Durán \& Oliva, 2014; Dar, Vignoles, Rondeland \& Dreyfuss, 2015; Juhásová et al., 2016; Vásquez et al., 2016; van Paridon, Colwell, Goater \& Gilleard, 2017; Criscione, van Paridon, Gilleard \& Goater, 2020) have so far been published. The above studies have shown that microsatellites, as molecular population markers, are more discriminative than rDNA and mtDNA. They have also demonstrated their usefulness in revealing the population genetic structure and historical migratory routes of parasites with a wide distribution range (Śtefka et al., 2009; Juhásová et al., 2016; Bazsalovicsová et al., 2020), and also migratory patterns of their hosts (Criscione et al., 2006). However, prior to our study, no population genetic studies utilizing microsatellites had been performed on monogeneans. Even though the primer set for the amplification of microsatellite loci was designed for Gyrodactylus (Faria et al., 2011), their sole application was to assess modes of reproduction in this genus (Schelkle, Faria, Johnson, Oosterhout \& Cable, 2012). The main reason for the scarcity of published microsatellite studies on monogeneans (and population markers in general) 
remains the collection of appropriate material for NGS. As the quantity and quality of the genomic DNA isolated from a small-sized parasite may be insufficient, one option is to pool a considerable number of specimens (Vanhove, Briscoe, Jorissen, Littlewood \& Huyse, 2018). However, considering that monogeneans are generally small-sized and that correct species identification is rather difficult without magnifying optical methods, there is a high risk of cross-species contamination in the pooled samples, rendering the obtained genomic data challenging to process. The pooling technique was employed in this study because it used morphologically easily identifiable monogenean species. Therefore, we were able to provide and utilize the first set of de novo developed microsatellite markers that might open possibilities for population-genetic studies in dactylogyrid monogeneans. Particularly in the case of this highly diversified group of parasites, which often exhibit extremely narrow host specificity, their genetic population variability might reflect the remarkable variability of their hosts.

\section{Acknowledgements}

We are grateful to Kateřina Čermáková, Jaroslav Červenka, Milan Gelnar, Maria Lujza Červenka Kičinja, Tomáš Pakosta, Eva Řehulková and Petra Zahradníčková (Masaryk University, Brno, Czech Republic) for their help with fish dissection and parasite collection, and Kristýna Hejlová for help with laboratory work. We are also indebted to Jasná Vukić (Charles University, Prague, Czech Republic) and Radek Šanda (National Museum, Prague, Czech Republic) for fish collection, and to Stefano Porcelloti (Arezzo, Italy), Dario Marić (Dobrič b. b., Bosnia and Herzegovina), Ivan Bogut (Josip Jujar Strossmayer University of Osijek, Croatia), Ivana Buj and Zoran Marčić (University of Zagreb, Croatia), and Stamatis Zogaris (Hellenic Centre for Marine Research, Greece) for arranging fishing permits and for their help with field work. We kindly thank Peter Mikulíček and Peter Papežík for discussion regarding data interpretation, and Matthew Nicholls for English revision of the final draft. The study was funded by the Czech Science Foundation (project number GA20-13539S).

\section{Author Contribution}

AŠ supervised, and together with LG designed the study. A $\check{S}$ and MB processed fish and collected the parasites. LG designed primers and protocols and performed laboratory protocols. LG and MB analyzed the genomic data. MB performed all molecular and statistical analyses. MB interpreted the results and wrote the manuscript with contributions from AŠ. All authors read and approved the final version of the manuscript.

\section{Data Availability}

The microsatellite genotypes data that are accessible in the Dryad data repository (doi:XXX).

\section{Tables and Figures}

Figure 1. Map of collection sites in the Apennine Peninsula and the Balkans

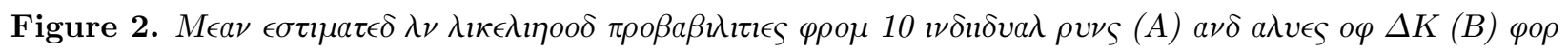

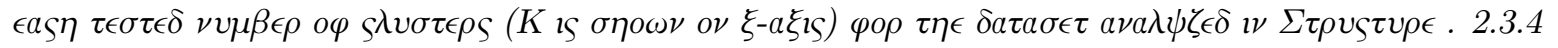

Figure 3. Results of Bayesian clustering analyses

Only bar plots for the estimated number of clusters $(\mathrm{K}) 3,5$, and 8 are shown. Each horizontal line within brackets represents one of 159 analyzed individuals. The estimated proportion of membership to a particular cluster is indicated by different colors. Labels on the right indicate collection sites of particular individuals.

Figure 4. Results of PCoA computed in GenAlEx v. 6.5 for all analyzed populations (A), and for populations representing sites $B 1-B 3$, and $C 1-C 3$ only $(B)$

Colored shapes indicate sites of collection of particular individuals. Percentage of variation explained by axes is presented in Table 6 . 
Table 1. List of host species investigated for presence of Dactylogyrus vistulae individuals including their respective collection sites with coordinates

Codes labeled as ID are used in the following tables and figures. $\mathrm{N}=$ number of collected and analyzed $D$. vistulae individuals from the respective host.

Table 2. Description of de novo developed microsatellites markers for D. vistulae and conditions for multiplexing into five PCR sets

Table 3. Summary of basic population-genetic statistical parameters for 24 microsatellite loci by collection site

TNA $=$ total number of alleles of locus; $\mathrm{Na}=$ number of alleles at particular site; $\mathrm{H}_{\mathrm{O}}=$ observed heterozygosity; $\mathrm{H}_{\mathrm{E}}=$ estimated heterozygosity; I1a = data only for $D$. vistulae from $S$. squalus ; I1b = data only for D. vistulae from T. muticellus .

Table 4. Descriptive statistics indicating mean values for basic parameters for all loci by each collection site

$\mathrm{N}=$ total number of alleles; $\mathrm{Na}=$ number of alleles per individual; $\mathrm{Ne}=$ effective number of alleles; I = Shannon's diversity index; $\mathrm{H}_{\mathrm{O}}=$ observed heterozygosity; $\mathrm{H}_{\mathrm{E}}=$ estimated heterozygosity; $\mathrm{F}=$ fixation index; I1a = data only for $D$. vistulae from $S$. squalus ; I1b = data only for $D$. vistulae from $T$. muticellus

Table 5. Pair-wise Nei-distances and $F_{S T}$ between individuals from each population pair

Table 6. Percentage of variation explained by the first three axes using PCoA on microsatellite markers in Figure 4

\section{References}

Abell, R., Thieme, M. L., Revenga, C., Bryer, M., Kottelat, M., Bogutskaya, N., .. Petry, P. (2008). Freshwater ecoregions of the world: A new map of biogeographic units for freshwater biodiversity conservation. BioScience , 58, 403-414.

Albrecht, C., \& Wilke, T. (2008). Ancient Lake Ohrid: Biodiversity and evolution. Hydrobiologia , 615, $103-140$.

Antonio Baeza, J., Sepúlveda, F. A., \& Teresa González, M. (2019). The complete mitochondrial genome and description of a new cryptic species of Benedenia Diesing, 1858 (Monogenea: Capsalidae), a major pathogen infecting the yellowtail kingfish Seriola lalandi Valenciennes in the South-East Pacific. Parasites 83 Vectors $, 12,490$.

Aydogdu, A., Altunel, F. N., \& Yildirimhan, H. S. (2001). Occurence of helminths in Chub, Leuciscus cephalus , of the Doganci (Bursa) dam lake, Turkey. Bulletin of the European Association of Fish Pathologists , 21, $246-251$

Bazsalovicsová, E., Králová-Hromadová, I., Juhásová, L., Mikulíček, P., Oravcová, A., Minárik, G., \& Štefka, J. (2020). Comparative analysis of monozoic fish tapeworms Caryophyllaeus laticeps (Pallas, 1781) and recently described Caryophyllaeus chondrostomi Barčák, Oros, Hanzelová, Scholz, 2017, using microsatellite markers.Parasitology Research, 119, 3995-4004.

Benovics, M., Desdevises, Y., Vukić, J., Šanda, R., \& Šimková, A. (2018). The phylogenetic relationships and species richness of host-specific Dactylogyrus parasites shaped by the biogeography of Balkan cyprinids. Scientific Reports , 8, 13006.

Benovics, M., Desdevises, Y., Šanda, R., Vukić, J., Scheifler, M., Doadrio, I., ... Šimková, A. (2020a). High diversity of fish ectoparasitic monogeneans (Dactylogyrus ) in the Iberian Peninsula: a case of adaptive radiation? Parasitology, 147, 418-430. 
Benovics, M., Desdevises, Y., Šanda, R., Vukić, J., \& Šimková, A. (2020b). Cophylogenetic relationships between Dactylogyrus (Monogenea) ectoparasites and endemic cyprinoids of the north-eastern European periMediterranean region. Journal of Zoological Systematics and Evolutionary Research , 58, 1-21.

Benovics, M., Francová, K., Volta, P., Dlapka, V., \& Šimková, A. (2021). Helminth communities of endemic cyprinoids of the Apennine Peninsula, with remarks on ectoparasitic monogeneans, and a description of four new Dactylogyrus Diesing, 1850 species. Parasitology , 2021, 1-16.

Bianco, P. G. (1990). Potential role of the paleohistory of the Mediterranean and Parathethys basins on the early dispersal of Euro Mediterranean freshwater fishes. Ichthyological Exploration of Freshwaters , 1, $167-184$.

Bordon, A., Peyron, O., Lezine, A. M., Brewer, S., \& Fouache, E. (2009). Pollen-inferred Late-Glacial and Holocene climate in southern Balkans (Lake Maliq). Quaternary International, 200, 19-30.

Buj, I., Vukić, J., Šanda, R., Perea, S., Ćaleta, M., Marčic, Z., ... Mrakovčić M. (2010) Morphological comparison of bleaks (Alburnus, Cyprinidae) from the Adriatic Basin with the description of a new species. Folia Zoologica , 59, 129-141.

Buj, I., Marčić, Z., Čaleta, M., Šanda, R., Geiger, M. F., Freyhof, J., ... Vukić, J. (2017). Ancient connections among European rivers and watersheds revealed from the evolutionary history of the genus Telestes (Actinopterygii; Cypriniformes). PLoS ONE , 12, e0187366.

Criscione, C. D., \& Blouin, M. S. (2006). Minimal selfing, few clones, and no among-host genetic structure in a hermaphroditic parasite with asexual larval propagation. Evolution , 60, 553-562.

Criscione, C. D., Cooper, B., \& Blouin, M. S. (2006). Parasite genotypes identify source populations of migratory fish more accurately than fish genotypes. Ecology , 87, 823-828.

Criscione, C. D., Vilas, R., Paniagua, E., \& Blouin, M. S. (2011). More than meets the eye: detecting cryptic microgeographic population structure in a parasite with a complex life cycle. Molecular Ecology , 20, 2510-2524.

Criscione, C. D., van Paridon, B. J., Gilleard, J. S., \& Goater, C. P. (2020). Clonemate cotransmission supports a role for kin selection in a puppeteer parasite. PNAS , 117, 5970-5976.

Dar, Y., Vignoles, P., Rondelaud, D., \& Dreyfuss, G. (2015). Role of lymnaeid snail Pseudosuccinea collumnela on the transmission of the liver fluke Fasciola hepatica in Egypt. Journal of Helminthology , 89, 699-706.

Doadrio, I. (1990). Phylogenetic relationships and classification of western Palearctic species of the genus Barbus (Osteichthyes, Cyprinidae). Aquatic Living Resources , 3, 265-282.

Earl, D. A., \& vonHoldt, B. M. (2012). STRUCTURE HARVESTER: a website and program for visualizing STRUCTURE output and implementing the Evanno method. Conservation Genetic Resources , 4, 359-361.

Evanno, G., Regnaut, S., \& Gould, J. (2005). Detecting the number of clusters of individuals using the software STRUCTURE: a simulation study. Molecular Ecology , 14, 2611-2620.

Falush, D., Stephens, M., \& Pritchard, J. K. (2007). Inference of population structure using multilocus genotype data: dominant markers and null alleles. Molecular Ecology Notes , 7, 574-578.

Faria, P. J., Lazarus, C. M., van Oosterhout, C., Harris, P. D., \& Cable, J. (2011). First polymorphic microsatellites for the gyrodactylids (Monogenea), an important group of fish pathogens. Conservation Genetics Resources , 3, 177-180.

Geiger, M. F., Herder, F., Monaghan, M. T., Almada, V., Barbieri, R., Bariche, M., .. Freyhof J. (2014). Spatial heterogeneity in the Mediterranean biodiversity hotspot affects barcoding accuracy of its freshwater fishes. Molecular Ecology Resources , 14, 1210-1221. 
Gibson, D. I., Timofeeva, T. A., \& Gerasev, P. I. (1996). Catalogue of the nominal species of the monogeneans of genus DactylogyrusDiesing, 1850 and their host genera. Systematic Parasitology , 35, 3-48.

Gower, C. M., Gabrielli, A. F., Sacko, M., Dembelé, R.., Golan, R., Emery, A. M., .. Webster, J. P. (2011). Population genetics of Schistosoma haematobium : development of novel microsatellite markers and their application to schistosomiasis control in Mali.Parasitology , 138, 978-994.

Hoffman, G. L. (1999). Parasites of North American freshwater fishes (2nd ed). Ithaca: Cornell University Press.

Huyse, T., \& Volckaert, F. A., (2002). Identification of a host-associated species complex using molecular and morphometric analyses, with the description of Gyrodactylus rugiensoides n. sp. (Gyrodactylidae, Monogenea). International Journal for Parasitology, 32, 907-919.

Huyse, T., Oeyen, M., Larmuseau, M. H. D., \& Volckaert, F. A. M. (2017). Co-phylogeographic study of the flatworm Gyrodactylus gondae and its goby host Pomatoschistus minutus .Parasitology International , 66, $119-125$.

Juhásová, L., Králová-Hromadová, I., Bazsalovicsová, E., Minárik, G., Štefka, J., Mikulíček, P., .. Pybus, M. (2016). Population structure and dispersal routes of an invasive parasite Fascioloides magna, in North America and Europe. Parasites \& Vectors , 9, 547.

Khang, T. F., Soo, O. Y. M., Tan, W. B., \& Lim, L. H. S. (2016). Monogenean anchor morphometry: systematic value, phylogenetic signal, and evolution. PeerJ , 4, e1668.

Kmentová, N., Gelnar, M., Mendlová, M., Van Steenberge, M., Koblmüller, S., \& Vanhove, M. P. M. (2016). Reduced host-specificity in a parasite infecting non-littoral Lake Tanganyika cichlids evidenced by intraspecific morphological and genetic diversity. Scientific Reports , 6, 39605.

Kmentová, N., Koblmüller, S., Van Steenberge, M., Raeymaekers, J. A. M., Artois, T., De Keyzer, E. L. R., ... Vanhove, M. P. M. (2020a) Weak population structure and recent demographic expansion of the monogenean parasite Kapentagyrus spp. infecting clupeid fishes of Lake Tanganyika, East Africa. International Journal for Parasitology , 50, 471-486,

Kmentová, N., Koblmüller, S., Van Steenberge, M., Artois, T., Muterezi Bukinga, F., Mulimbwa N'sibula, T., ... Vanhove, M. P. M. (2020b). Failure to diverge in African Great Lakes: the case ofDolicirroplectanum lacustre gen. nov., comb. nov. (Monogenea, Diplectanidae) infecting latid hosts. Journal of Great Lakes Research , 46, 1113-1130.

Kopelman, N. M., Mayzel, J., Jakobsson, M., Rosenberg, N. A., \& Mayrose, I. (2015). CLUMPAK: a program for identifying clustering modes and packaging population structure inferences across K". Molecular Ecology Resources , 15, 1179-1191.

Koskivaara, M., \& Valtonen, E. T. (1992). Dactylogyrus(Monogenea) communities on the gills of roach in three lakes in Central Finland. Parasitology, 104, 263-272.

Kottelat, M., \& Freyhof, J. (2007). Handbook of European freshwater fishes . Berlin: Kottelat, Cornol and Freyhof.

Kuchta, R., Řehulková, E., Francová, K., Scholz, T., Morand, S., \& Šimková, A. (2020). Diversity of monogenans and tapeworms in cypriniform fishes across two continents. International Journal for Parasitology , $50,771-786$.

Louhi, K.-R., Karvonen, A., Rellstab, C., \& Jokela, J. (2010). Is the population genetic structure of complex life cycle parasites determined by the geographic range of the most motile host? Infections, Genetics and Evolution , 10, 1271-1277.

Luo, H. Y., Nie, P., Zhang, Y. A., Yao, W. J., \& Wang, G. T. (2003). Genetic differentiation in populations of the cestodeBothriocephalus acheilognathi (Cestoda, Pseudophyllidea) as revealed by eight microsatellite 
markers. Parasitology , 126, 493-501.

Mariniello, L., Ortis, M., D’Amelio, S., \& Petrarca, V. (2004). Morphometric variability between and within species of LigophorusEuzet \& Suriano, 1977 (Monogenea: Ancyrocephalidae) in the Mediterranean Sea. Systematic Parasitology , 57, 183-190.

Meglécz, E., Costedoat, C., Dubut, V., Gilles, A., Malausa, T., Pech, N., \& Martin, J. F. (2010). QDD: a user-friendly program to select microsatellite markers and design primers from large sequencing projects. Bioinformatics , 26: 403-404.

Mhaisen, F. T., \& Abdul-Ameer, K. N. (2019). Checklists ofDactylogyrus species (Monogenea) from Fishes of Iraq.Biological and Applied Environmental Research , 3, 1-36.

Moravec, F. (2001) Checklist of the metazoan parasites of fishes of Czech Republic and Slovak Republic (1873-2000) . Prague: Academia.

Palandačić, A., Bravničar, J., Zupančič, P., Šanda, R., \& Snoj, A. (2015). Molecular data suggest a multispecies complex of Phoxinus(Cyprinidae) in the Western Balkan Peninsula. Molecular Phylogenetics and Evolution , 92, 118-123.

Peakall, R., \& Smouse, P. E. (2006). GenAlEx 6: genetic analysis in Excel. Population genetic software for teaching and research.Molecular Ecology Notes , 6, 288-295.

Peakall, R., \& Smouse P. E. (2012). GenAlEx 6.5: genetic analysis in excel. Population genetic software for teaching and research - an update. Bioinformatics , 28, 2537-2539

Perea, S., Böhme, M., Zupančič, P., Freyhof, J., Šanda, R., Özuluğ, M., .. Doadrio, I. (2010). Phylogenetic relationships and biogeographical patterns in Circum-Mediterranean subfamily Leuciscinae (Teleostei, Cyprinidae) inferred from both mitochondrial and nuclear data. BMC Evolutionary Biology , $10,265$.

Pettersen, R. A., Junge, C., Østbye, K., Mo, T. A., \& Vøllestad, L. A. (2021). Genetic population structure of the monogenean parasiteGyrodactylus thymalli and its host European grayling (Thymallus thymallus ) in a large Norwegian lake.Hydrobiologia , 848, 547-561.

Pielou, E. C. (1979). Biogeography. New York: Wiley.

Pritchard, J. K., Stephens, M., \& Donnelly, P. J. (2000). Inference of population structure using multilocus genotype data. Genetics , 155, 945-959.

Pugachev, O. N., Gerasev, P. I., Gussev, A. V., Ergens, R., \& Khotenowsky, I. (2009). Guide to Monogenoidea of freshwater fish of Palearctic and Amur Regions. Milan: Ledizione-Ledi Publishing.

Rahmouni, C., Van Steenberge, M., Vanhove, M. P. M., \& Šimková, A. (2020). Intraspecific morphological variation in Cichlidogyrus (Monogenea) parasitizing two cichlid hosts from Lake Tanganyika exhibiting different dispersal capacities. Hydrobiologia (2020) doi:10.1007/s10750-020-04429-1.

Řehulková, E., Seifertová, M., Přikrylová, I., \& Francová, K. (2018). Monogenea. In Scholz, T., Vanhove, M. P. M., Smit, N., Jayasundera, Z., \& Gelnar, M. (Eds.), A guide to the parasites of African freshwater fishes . (pp. 185-243). Brussels: AbcTaxa.

Řehulková, E., Benovics, M., \& Šimková, A. (2020). Uncovering the diversity of monogeneans (Platyhelminthes) on endemic cypriniform fishes of the Balkan Peninsula: new species of Dactylogyrus and comments on their phylogeny and host-parasite associations in a biogeographic context. Parasite , 27, 66.

Rodríguez-González, A., Míguez-Lozano, R., Llopis-Belenguer, C., \& Balbuena, J. A. (2015). Phenotypic plasticity in haptoral structures ofLigophorus cephali (Monogenea: Dactylogyridae) on the flathead mullet (Mugil cephalus ): a geometric morphometric approach.International Journal for Parasitology , 45, $295-303$.

Schelkle, B., Faria, P. J., Johnson, M. B., van Oosterhout, C., \& Cable, J. (2012). Mixed infections and hybridisation in monogenean parasites. PLoS ONE , 7, e39506. 
Seifertová, M., Vyskočilová, M., Morand, S., \& Šimková, A. (2008). Metazoan parasites of freshwater cyprinid fish (Leuciscus cephalus ): testing biogeography hypotheses of species diversity.Parasitology, 135, 1417-1435.

Schönhuth, S., Vukić, J., Šanda, R., Yang, L., \& Mayden, R. L. (2018). Phylogenetic relationships and classification of the Holarctic family Leuciscidae (Cypriniformes: Cyprinoidei). Molecular Phylogenetics and Evolution , 127, 781-799.

Schultheiss, R., Albrecht, C., Bossneck, U., \& Wilke, T. (2008). The neglected side of speciation in ancient lakes: Phylogeography of an in conspicuous mollusc taxon in lakes Ohrid and Prespa.Hydrobiologia , 615, $141-156$.

Šimková, A., Morand, S., Jobet, E., Gelnar, M., \& Verneau, O. (2004). Molecular phylogeny of congeneric monogenean parasites (Dactylogyrus ): a case of intrahost speciation. Evolution , 58, 1001-1018.

Šimková, A., Verneau, O., Gelnar, M., \& Morand, S. (2006). Specificity and specialization of congeneric monogeneans parasitizing cyprinid fish.Evolution , 60, 1023-1037.

Šimková, A., Benovics, M., Rahmouni, I., \& Vukić, J (2017). Host-specific Dactylogyrus parasites revealing new insights on the historical biogeography of Northwest African and Iberian cyprinid fish. Parasites $\mathcal{E}^{3}$ Vectors , 10, 589 .

Stefani, F., Galli, P., Crosa, G., Zaccara, S., \& Calamari, D. (2004). Alpine and Apennine barriers determining the differentiation of the rudd (Scardinius erythrophthalmus L.) in the Italian peninsula.Ecology of Freshwater Fish , 13, 168-175.

Štefka, J., Hypša, V., \& Scholz, T. (2009). Interplay of host specificity and biogeography in the population structure of a cosmopolitan endoparasite: microsatellite study of Ligula intestinalis (Cestoda). Molecular Ecology , 18, 1187-1206.

Steininger, F. F., \& Rögl, F. (1984). Paleogeography and palinspastic reconstruction of the Neogene of the Mediterranean and Paratethys . In Dixon, J. E., \& Robertson, A. H. (Eds.), The geological evolution of the eastern Mediterranean . (pp. 659-668). Oxford: Blackwell.

Sušnik S., Snoj A., Wilson I. F., Mrdak D. \& Weiss S. (2007) Historical demography of brown trout (Salmo trutta) in the Adriatic drainage including the putative S. letnica endemic to Lake Ohrid.Molecular Phylogenetics and Evolution 44: 63-76.

Tan, M., \& Armbruster, J. W. (2018). Phylogenetic classification of extant genera of fishes of the order Cypriniformes (Teleostei: Ostariophysi). Zootaxa , 4476, 006-039.

Umhang, G., Grenouillet, F., Bastid, V., M'Rad, S., Balot, B., Oudni-M'Rad, M., .. B Boué, F. (2018). Investigating the genetic diversity of Echinococchus granulosus sensu stricto with new microsatellites. Parasitology Research , 117, 2743-2755.

Valdivia, I. M., Criscione, C. D., Cárdenas, L., Durán, C. P., \& Oliva, M. E. (2014). Does a facultative precocious life cycle predispose the marine trematode Proctoeces cf. lintoni to inbreeding and genetic differentiation among host species? International Journal for Parasitology , 44, 183-188.

Vignon, M., \& Sasal, P. (2010). The use of geometric morphometrics in understanding shape variability of sclerotized haptoral structures of monogeneans (Platyhelminthes) with insights into biogeographic variability. Parasitology International , 59, 183-191.

Vanhove, M. P. M., Briscoe, A. G., Jorissen, M. W. P., Littlewood, D. T. J., \& Huyse, T. (2018). The first next-generation sequencing approach to the mitochondrial phylogeny of African monogenean parasites (Platyhelminthes: Gyrodactylidae and Dactylogyridae). BMC Genomics , 19, 520.

van Paridon, B. J., Colwell, D. D., Goater, C. P., \& Gilleard, J. S. (2017). Population genetic analysis informs the invasion history of the emerging trematode Dicrocoelium dendriticum into Canada.International Journal for Parasitology , 47, 845-856. 
Vásquez, A. A., Lounnas, M., Sánchez, J., Alba, A., Milesi, A., \& Hurtrez-Bousséz, S. (2016). Genetic and infective diversity of the liver fluke Fasciola hepatica (Trematoda: Digenea) from Cuba. Journal of Helminthology , 90, 719-725.

Wagner, B., \& Wilke, T. (2011). Evolutionary and geological history of the Balkan lakes Ohrid and Prespa. Biogeosciences , 8, 995-998.

Waelbroeck, C., Labeyrie, L., Michel, E., Duplessy, J. C., McManus, J. F., Lambeck, K. ... Labracherie, M. (2002). Sea-level and deep water temperature changes derived from benthic foraminifera isotopic records.Quaternary Science Reviews , 21, 295-305.

\begin{tabular}{llllll}
\hline ID & Host species & N & Country & Locality & coordinates \\
\hline B1 & Chondrostoma phoxinus & 5 & Bosnia & Šujica, Šujičko Polje & $43^{\circ} 49^{\prime} 41.43^{\prime \prime} \mathrm{N} 17^{\circ} 10^{\prime} 4$ \\
B2 & Phoxinellus alepidotus & 5 & Bosnia & Bosansko Grahovo, Korana river & $44^{\circ} 10^{\prime} 37.00^{\prime \prime} \mathrm{N} 16^{\circ} 23^{\prime} 0$ \\
B3 & Phoxinellus pseudalepidotus & 7 & Bosnia & Lištica, Polog & $43^{\circ} 20^{\prime} 32.09^{\prime \prime} \mathrm{N} 17^{\circ} 41^{\prime} 3$ \\
B4 & Squalius tenellus & 32 & Bosnia & Šujica & $43^{\circ} 42^{\prime} 05.07^{\prime \prime} \mathrm{N} 17^{\circ} 15^{\prime} 5$ \\
C1 & Squalius illyricus & 7 & Croatia & Cetina river, Kosore & $43^{\circ} 56^{\prime} 29.78^{\prime \prime} \mathrm{N} 16^{\circ} 26^{\prime} 2$ \\
C2 & Squalius svallize & 6 & Croatia & Konavočica, Grude & $42^{\circ} 31^{\prime} 33.86^{\prime \prime} \mathrm{N} 18^{\circ} 22^{\prime} 0$ \\
C3 & Telestes turskyi & 30 & Croatia & rieka Čikola & $43^{\circ} 48^{\prime} 22.09^{\prime \prime} \mathrm{N} 16^{\circ} 17^{\prime} 2$ \\
C4 & Telestes croaticus & 10 & Croatia & medzi Sveti Rok a Ličko Cerje, rieka Obsenica & $44^{\circ} 21^{\prime} 03.64^{\prime \prime} \mathrm{N} 15^{\circ} 40^{\prime} 4$ \\
C5 & Telestes fontinalis & 12 & Croatia & Krbavsko polje, Laudonov gaj & $44^{\circ} 38^{\prime} 14.33^{\prime \prime} \mathrm{N} 15^{\circ} 40^{\prime} 0$ \\
G1 & Squalius prespensis & 11 & Greece & Aoos, Kalithea & $40^{\circ} 01^{\prime} 16.67^{\prime \prime} \mathrm{N} 20^{\circ} 41^{\prime} 4$ \\
G2 & Squalius peloponensis & 18 & Greece & Pamisos, Vasiliko & $37^{\circ} 15^{\prime} 17.39^{\prime \prime} \mathrm{N} 21^{\circ} 53^{\prime} 4$ \\
I1a & Squalius squalus & 5 & Italy & Torrente Cerfone, Intoppo & $43^{\circ} 26^{\prime} 12.03^{\prime \prime} \mathrm{N} 11^{\circ} 58^{\prime} 3$ \\
I1b & Telestes muticellus & 11 & Italy & Torrente Cerfone, Intoppo & $43^{\circ} 26^{\prime} 12.03^{\prime \prime} \mathrm{N} 11^{\circ} 58^{\prime} 3$ \\
\hline
\end{tabular}

Table 1. List of host species investigated for presence of Dactylogyrus vistulae individuals including their respective collection sites with coordinates

Codes labeled as ID are used in the following tables and figures. $\mathrm{N}=$ number of collected and analyzed $D$. vistulae individuals from the respective host.

Table 2. Description of de novo developed microsatellites markers for Dactylogyrus vistulae and conditions for multiplexing into five PCR sets

\begin{tabular}{|c|c|c|c|c|}
\hline Locus & Primer sequence $\left(5^{\prime}-3^{\prime}\right)$ & Repeat motif & Multiplex PCR sets & Multiplex PCR sets \\
\hline & & & Set & $\mu \mu$ \\
\hline \multirow[t]{2}{*}{ Dacty34 } & F: AGACATGCTCTGCTCACGAT & $(\mathrm{AT})_{9}$ & 1 & 0.15 \\
\hline & R: TGATTCTAAGATGCGGGCAC & & & \\
\hline \multirow[t]{2}{*}{ Dacty36 } & F: CCGGTTCTTAGTTTAATGGGC & $(\mathrm{AG})_{9}$ & 2 & 0.1 \\
\hline & R: TGTGCACTGTTCCATCATGT & & & \\
\hline \multirow[t]{2}{*}{ Dacty38 } & F: CAAGTGGACTCGCAACAGAC & $(\mathrm{AAT})_{8}$ & 1 & 0.15 \\
\hline & R: CGGCACAATGAAAGGCTAT & & & \\
\hline \multirow[t]{2}{*}{ Dacty 44} & F: ACACGAGATGGTGCTTGATG & $(\mathrm{AG})_{10}$ & 2 & 0.1 \\
\hline & R: TACGCTACAAGTGCTACAAGGA & & & \\
\hline \multirow[t]{2}{*}{ Dacty50 } & F: GCCCACGCTTGTCTTAACAT & $(\mathrm{AG})_{12}$ & 1 & 0.15 \\
\hline & R: GTATGTCAACGCGCTCAATG & & & \\
\hline \multirow[t]{2}{*}{ Dacty52 } & F: AACACCATGAGAATGGAGCG & $(\mathrm{AG})_{8}$ & 4 & 0.1 \\
\hline & R: AATAGAGGGAGGGAAGGTGG & & & \\
\hline \multirow[t]{2}{*}{ Dacty 54} & F: CTTCCAAGGGACAACAGGAG & $(\mathrm{AG})_{7}$ & 3 & 0.15 \\
\hline & R: TTGTCGATTTCAGCTCATGG & & & \\
\hline
\end{tabular}




\begin{tabular}{|c|c|c|c|c|}
\hline Locus & Primer sequence $\left(5^{\prime}-3^{\prime}\right)$ & Repeat motif & Multiplex PCR sets & Multiplex PCR sets \\
\hline Dacty 64 & $\begin{array}{l}\text { F: AGACCAGCAAACGAAGTTGG } \\
\text { R: TTGGTCATTGCTAAGGTTTCC }\end{array}$ & $(\mathrm{AG})_{7}$ & 3 & 0.35 \\
\hline Dacty65 & $\begin{array}{l}\text { F: TTGCATTGCGTGATGGAC } \\
\text { R: TTGTACGTGTTGGTGCGATT }\end{array}$ & $(\mathrm{AC})_{7}$ & 4 & 0.25 \\
\hline Dacty66 & $\begin{array}{l}\text { F: TGCAGCATCGATTAAGTCTCA } \\
\text { R: CCACTTGCATTCCCAGCTA }\end{array}$ & $(\mathrm{AG})_{7}$ & 2 & 0.15 \\
\hline Dacty67 & $\begin{array}{l}\text { F: TCATGAAAGAGAACGAAACGAA } \\
\text { R: TGGGTCAGACTGGATTTCCT }\end{array}$ & $(\mathrm{AT})_{7}$ & 2 & 0.4 \\
\hline Dacty68 & $\begin{array}{l}\text { F: AGGCATTTGCAACTCGATTA } \\
\text { R: GCCAATCGCTGAGTTTGAA }\end{array}$ & $(\mathrm{AGG})_{7}$ & 3 & 0.35 \\
\hline Dacty69 & $\begin{array}{l}\text { F: TAGAGGGAAGGCAAGTGTCC } \\
\text { R: GCCATAGAAGCCAGCGAA }\end{array}$ & $(\mathrm{AAAC})_{7}$ & 3 & 0.15 \\
\hline Dacty70 & $\begin{array}{l}\text { F AATGCTGCCGAATTAACAGG } \\
\text { R: TTGAGTGGGCTAGGTGTAGAAA }\end{array}$ & $(\mathrm{ATC})_{7}$ & 3 & 0.25 \\
\hline Dacty 73 & $\begin{array}{l}\text { F: AATTGAAGCGCTCCTCCG } \\
\text { R: TCAATATCCAGTCTCGCAGC }\end{array}$ & $(\mathrm{AG})_{7}$ & 4 & 0.15 \\
\hline Dacty 75 & $\begin{array}{l}\text { F CATGACCATGACAACCAACG } \\
\text { R: ATGCACCACGCATCTATTTG }\end{array}$ & $(\mathrm{CG})_{6}$ & 5 & 0.3 \\
\hline Dacty79 & $\begin{array}{l}\text { F: GCAGTTTGTCCTGGCATTTC } \\
\text { R: CACCAACTCGCCCTATGAGT }\end{array}$ & $(\mathrm{AT})_{6}$ & 4 & 0.2 \\
\hline Dacty 84 & $\begin{array}{l}\text { F: AAGGTTGTAGCCTTGGTCAATC } \\
\text { R: CAGCCAGTTGATCATCAGTTC }\end{array}$ & $(\mathrm{AG})_{6}$ & 5 & 0.2 \\
\hline Dacty 85 & $\begin{array}{l}\text { F: GGTCGACGCTTCTCTTTGAT } \\
\text { R: GTCTCTAGAATTCGCCCGGA }\end{array}$ & $(\mathrm{AG})_{6}$ & 1 & 0.3 \\
\hline Dacty 87 & $\begin{array}{l}\text { F: AAACATAGCCGCCAACCAG } \\
\text { R: TGTACACGAGCATTGAAGAGC }\end{array}$ & $(\mathrm{AGC})_{6}$ & 5 & 0.15 \\
\hline Dacty92 & $\begin{array}{l}\text { F: CTTGCTTCAAACTCGGCTGT } \\
\text { R: CATGCATTCCCATCATTCAC }\end{array}$ & $(\mathrm{AAGGT})_{6}$ & 3 & 0.2 \\
\hline Dacty93 & $\begin{array}{l}\text { F: ATTTGCCAATCTGTGCATGA } \\
\text { R: GGGTTGGGTTGTTGGTAAAGT }\end{array}$ & $(\mathrm{ACTG})_{6}$ & 4 & 0.25 \\
\hline Dacty96 & $\begin{array}{l}\text { F: GGACAAGTTGAGTTGCTCGG } \\
\text { R: GCGATACCATGTAGGGCAAG }\end{array}$ & $(\mathrm{AGC})_{6}$ & 5 & 0.25 \\
\hline Dacty99 & $\begin{array}{l}\text { F: AACATGGAATAGGAGTGGGC } \\
\text { R: TCAATTGTACACGGACGAGC }\end{array}$ & $(\mathrm{AAC})_{6}$ & 1 & 0.15 \\
\hline
\end{tabular}

Table 3. Summary of basic population-genetic statistical parameters for 24 microsatellite loci by collection site.

\begin{tabular}{llllllllllllllllll}
\hline & & & B1 & B1 & B1 & B2 & B2 & B2 & B3 & B3 & B3 & B4 & B4 & B4 & C1 & C1 \\
\hline Locus & Size (bp) & TNA & Na & Ho & He & Na & Ho & He & Na & Ho & He & Na & Ho & He & Na & Ho \\
Dacty34 & $204-218$ & 6 & 1 & - & - & 1 & - & - & 1 & - & - & 1 & - & - & 1 & - \\
Dacty36 & $179-211$ & 9 & 1 & - & - & 1 & - & - & 1 & - & - & 1 & - & - & 1 & - \\
Dacty38 & $231-240$ & 4 & 1 & - & - & 1 & - & - & 1 & - & - & 1 & - & - & 1 & - \\
Dacty44 & $226-252$ & 10 & 1 & - & - & 1 & - & - & 1 & - & - & 1 & - & - & 1 & - \\
Dacty50 & $90-110$ & 6 & 1 & - & - & 1 & - & - & 1 & - & - & 2 & - & 0.342 & 1 & - \\
Dacty52 & $134-158$ & 10 & 1 & - & - & 1 & - & - & 1 & - & - & 1 & - & - & 2 & 0.143 \\
Dacty54 & $186-212$ & 10 & 1 & - & - & 1 & - & - & 2 & - & - & 1 & - & - & 1 & - \\
Dacty64 & $245-253$ & 5 & 1 & - & - & 1 & - & - & 1 & - & - & 1 & - & - & 1 & - \\
Dacty65 & $215-239$ & 9 & 1 & - & - & 1 & - & - & 1 & - & - & 1 & - & - & 1 & -
\end{tabular}




\begin{tabular}{llllllllllllllllll}
\hline & & & B1 & B1 & B1 & B2 & B2 & B2 & B3 & B3 & B3 & B4 & B4 & B4 & C1 & C1 \\
\hline Dacty66 & $160-200$ & 13 & 1 & - & - & 1 & - & - & 1 & - & - & 2 & - & 0.219 & 1 & - \\
Dacty67 & $105-109$ & 3 & 1 & - & - & 1 & - & - & 1 & - & - & 1 & - & - & 1 & - \\
Dacty68 & $92-119$ & 8 & 1 & - & - & 1 & - & - & 1 & - & - & 1 & - & - & 1 & - \\
Dacty69 & $109-133$ & 6 & 1 & - & - & 1 & - & - & 2 & 0.143 & 0.133 & 2 & - & 0.404 & 1 & - \\
Dacty70 & $148-163$ & 5 & 1 & - & - & 1 & - & - & 1 & - & - & 1 & - & - & 1 & - \\
Dacty73 & $108-148$ & 16 & 4 & - & 0.720 & 1 & - & - & 2 & - & - & 5 & 0.125 & 0.561 & 1 & - \\
Dacty75 & $198-204$ & 2 & 1 & - & - & 1 & - & - & 2 & 0.143 & 0.133 & 1 & - & - & 1 & - \\
Dacty79 & $189-203$ & 4 & 1 & - & - & 1 & - & - & 1 & - & - & 1 & - & - & 1 & - \\
Dacty84 & $143-175$ & 8 & 1 & - & - & 2 & - & 0.320 & 1 & - & - & 1 & - & - & 1 & - \\
Dacty85 & $181-196$ & 5 & 1 & - & - & 1 & - & - & 1 & - & - & 1 & - & - & 1 & - \\
Dacty87 & $92-107$ & 6 & 1 & - & - & 1 & - & - & 1 & - & - & 1 & - & - & 1 & - \\
Dacty92 & $126-151$ & 6 & 2 & - & 0.320 & 1 & - & - & 1 & - & - & 2 & 0.031 & 0.031 & 1 & - \\
Dacty93 & $110-122$ & 4 & 1 & - & - & 1 & - & - & 1 & - & - & 1 & - & - & 1 & - \\
Dacty96 & $174-189$ & 5 & 1 & - & - & 1 & - & - & 2 & 0.167 & 0.153 & 1 & - & - & 2 & - \\
Dacty99 & $185-203$ & 7 & 1 & - & - & 1 & - & - & 1 & - & - & 2 & - & 0.064 & 1 & - \\
\hline
\end{tabular}

Codes in the first row represent collection site IDs; TNA = total number of alleles of locus; $\mathrm{Na}=$ number of alleles at particular site; $\mathrm{H}_{\mathrm{O}}=$ observed heterozygosity; $\mathrm{H}_{\mathrm{E}}=$ estimated heterozygosity; I1a = data only for $D$. vistulae from $S$. squalus ; I1b = data only for D. vistulae from $T$. muticellus

Table 3 (continue). Summary of basic population-genetic statistical parameters for 24 microsatellite loci by collection site.

\begin{tabular}{|c|c|c|c|c|c|c|c|c|c|c|c|c|c|c|c|c|c|}
\hline $\mathrm{C} 4$ & $\mathrm{C} 4$ & C4 & C5 & C5 & C5 & G1 & G1 & G1 & G2 & G2 & G2 & I1 & I1 & I1 & I1a & I1a & I1a \\
\hline $\mathrm{Na}$ & Но & $\mathrm{He}$ & $\mathrm{Na}$ & Но & $\mathrm{He}$ & $\mathrm{Na}$ & Ho & $\mathrm{He}$ & $\mathrm{Na}$ & Но & $\mathrm{He}$ & $\mathrm{Na}$ & Но & $\mathrm{He}$ & $\mathrm{Na}$ & Но & $\mathrm{He}$ \\
\hline 1 & - & - & 1 & - & - & 4 & 0.667 & 0.667 & 1 & - & - & 3 & - & 0.461 & 2 & - & 0.320 \\
\hline 2 & - & 0.180 & 1 & - & - & 2 & 0.091 & 0.087 & 1 & - & - & 6 & 0.125 & 0.504 & 5 & 0.400 & 0.760 \\
\hline 1 & - & - & 1 & - & - & 3 & 0.545 & 0.483 & 1 & - & - & 2 & 0.063 & 0.061 & 2 & 0.200 & 0.180 \\
\hline 1 & - & - & 1 & - & - & 7 & 0.909 & 0.814 & 1 & - & - & 4 & 0.200 & 0.504 & 3 & 0.600 & 0.540 \\
\hline 1 & - & - & 1 & - & - & 1 & - & - & 2 & 0.056 & 0.054 & 2 & - & 0.430 & 1 & - & - \\
\hline 1 & - & - & 1 & - & - & 8 & 0.636 & 0.843 & 1 & - & - & 4 & - & 0.484 & 3 & - & 0.560 \\
\hline 1 & - & - & 1 & - & - & 6 & 0.909 & 0.793 & 1 & - & - & 4 & - & 0.484 & 3 & - & 0.560 \\
\hline 1 & - & - & 1 & - & - & 4 & 0.455 & 0.550 & 1 & - & - & 2 & 0.063 & 0.170 & 2 & 0.200 & 0.420 \\
\hline 1 & - & - & 1 & - & - & 7 & 0.727 & 0.756 & 1 & - & - & 1 & - & - & 1 & - & - \\
\hline 1 & - & - & 1 & - & - & 9 & 0.727 & 0.826 & 3 & 0.056 & 0.106 & 4 & 0.125 & 0.482 & 3 & 0.400 & 0.540 \\
\hline 1 & - & - & 2 & - & 0.153 & 3 & 0.125 & 0.570 & 2 & 0.111 & 0.346 & 2 & 0.125 & 0.219 & 2 & 0.400 & 0.480 \\
\hline 1 & - & - & 2 & - & 0.375 & 7 & 0.909 & 0.789 & 1 & - & - & 3 & 0.125 & 0.420 & 3 & 0.400 & 0.340 \\
\hline 1 & - & - & 1 & - & - & 4 & 0.364 & 0.318 & 3 & 0.444 & 0.647 & 2 & - & 0.430 & 1 & - & - \\
\hline 1 & - & - & 1 & - & - & 4 & 0.364 & 0.384 & 1 & - & - & 2 & 0.063 & 0.061 & 2 & 0.200 & 0.180 \\
\hline 2 & - & 0.420 & 3 & - & 0.569 & 4 & 0.273 & 0.566 & 1 & - & - & 5 & 0.063 & 0.494 & 4 & 0.200 & 0.660 \\
\hline 1 & - & - & 1 & - & - & 1 & - & - & 1 & - & - & 1 & - & - & 1 & - & - \\
\hline 1 & - & - & 1 & - & - & 3 & 0.455 & 0.574 & 1 & - & - & 2 & 0.063 & 0.061 & 1 & - & - \\
\hline 1 & - & - & 1 & - & - & 3 & 0.545 & 0.417 & 1 & - & - & 6 & 0.125 & 0.699 & 5 & 0.400 & 0.600 \\
\hline 1 & - & - & 1 & - & - & 2 & 0.364 & 0.463 & 2 & 0.250 & 0.305 & 1 & - & - & 1 & - & - \\
\hline 1 & - & - & 1 & - & - & 4 & 0.364 & 0.541 & 3 & - & 0.364 & 4 & 0.063 & 0.373 & 4 & 0.200 & 0.740 \\
\hline 1 & - & - & 1 & - & - & 2 & 0.364 & 0.397 & 1 & - & - & 4 & 0.188 & 0.484 & 3 & 0.600 & 0.560 \\
\hline 1 & - & - & 1 & - & - & 4 & 0.545 & 0.678 & 1 & - & - & 2 & - & 0.305 & 2 & - & 0.480 \\
\hline 1 & - & - & 1 & - & - & - & - & - & 2 & 0.235 & 0.360 & 3 & - & 0.461 & 2 & - & 0.320 \\
\hline 1 & - & - & 1 & - & - & 2 & 0.545 & 0.463 & 1 & - & - & 4 & 0.125 & 0.492 & 3 & 0.400 & 0.640 \\
\hline
\end{tabular}


$\mathrm{TNA}=$ total number of alleles of locus; $\mathrm{Na}=$ number of alleles at particular site; $\mathrm{H}_{\mathrm{O}}=$ observed heterozygosity; $\mathrm{H}_{\mathrm{E}}=$ estimated heterozygosity; I1a = data only for $D$. vistulae from $S$. squalus $; \mathrm{I} 1 \mathrm{~b}=$ data only for D. vistulae from T. muticellus.

Table 4. Descriptive statistics indicating mean values for basic parameters for all loci by each collection site.

\begin{tabular}{llllllll}
\hline Pop & $\mathrm{N}$ & $\mathrm{Na}$ & $\mathrm{Ne}$ & $\mathrm{I}$ & $\mathrm{Ho}$ & $\mathrm{He}$ & $\mathrm{F}$ \\
\hline $\mathrm{B} 1$ & 5 & 1.167 & 1.127 & 0.076 & 0.000 & 0.043 & 1.000 \\
$\mathrm{~B} 2$ & 5 & 1.042 & 1.020 & 0.021 & 0.000 & 0.013 & 1.000 \\
$\mathrm{~B} 3$ & 7 & 1.208 & 1.063 & 0.075 & 0.019 & 0.045 & 0.351 \\
$\mathrm{~B} 4$ & 32 & 1.375 & 1.119 & 0.115 & 0.007 & 0.068 & 0.794 \\
$\mathrm{C} 1$ & 7 & 1.083 & 1.035 & 0.039 & 0.006 & 0.024 & 0.788 \\
$\mathrm{C} 2$ & 6 & 1.042 & 1.016 & 0.019 & 0.000 & 0.012 & 1.000 \\
$\mathrm{C} 3$ & 30 & 1.250 & 1.050 & 0.049 & 0.001 & 0.023 & 0.939 \\
$\mathrm{C} 4$ & 10 & 1.083 & 1.039 & 0.039 & 0.000 & 0.025 & 1.000 \\
$\mathrm{C} 5$ & 12 & 1.167 & 1.088 & 0.075 & 0.000 & 0.046 & 1.000 \\
$\mathrm{G} 1$ & 10 & 3.917 & 2.662 & 0.966 & 0.453 & 0.499 & 0.083 \\
$\mathrm{G} 2$ & 18 & 1.417 & 1.171 & 0.153 & 0.048 & 0.091 & 0.424 \\
$\mathrm{I} 1 \mathrm{a}$ & 5 & 2.458 & 1.911 & 0.636 & 0.192 & 0.370 & 0.443 \\
$\mathrm{I} 1 \mathrm{~b}$ & 11 & 1.125 & 1.081 & 0.064 & 0.004 & 0.044 & 0.651 \\
\hline
\end{tabular}

Pop = collection site ID; $\mathrm{N}=$ total number of alleles; $\mathrm{Na}=$ number of alleles per individual; $\mathrm{Ne}=$ effective number of alleles; $\mathrm{I}=$ Shannon's diversity index; $\mathrm{H}_{\mathrm{O}}=$ observed heterozygosity; $\mathrm{H}_{\mathrm{E}}=$ estimated heterozygosity; F = fixation index; I1a = data only for D. vistulae from $S$. squalus ; I1b = data only for D. vistulae from T. muticellus .

Table 5. Pair-wise Nei-distances and $F_{S T}$ between individuals from each population pair.

\begin{tabular}{llllllllllllll}
\hline & B1 & B2 & B3 & B4 & C1 & C2 & C3 & C4 & C5 & G1 & G2 & I1a & I1b \\
\hline B1 & - & 0.231 & 0.226 & 0.263 & 0.195 & 0.194 & 0.415 & 0.939 & 0.890 & 0.467 & 0.819 & 0.354 & 0.815 \\
B2 & 0.031 & - & 0.281 & 0.224 & 0.103 & 0.527 & 0.619 & 0.959 & 0.909 & 0.487 & 0.850 & 0.377 & 0.854 \\
B3 & 0.068 & 0.084 & - & 0.197 & 0.281 & 0.278 & 0.364 & 0.850 & 0.809 & 0.477 & 0.765 & 0.356 & 0.768 \\
B4 & 0.069 & 0.057 & 0.086 & - & 0.207 & 0.270 & 0.369 & 0.882 & 0.834 & 0.494 & 0.806 & 0.383 & 0.748 \\
C1 & 0.033 & 0.005 & 0.085 & 0.059 & - & 0.322 & 0.442 & 0.946 & 0.897 & 0.482 & 0.843 & 0.363 & 0.834 \\
C2 & 0.022 & 0.045 & 0.083 & 0.079 & 0.048 & - & 0.664 & 0.958 & 0.905 & 0.487 & 0.849 & 0.373 & 0.855 \\
C3 & 0.055 & 0.060 & 0.117 & 0.120 & 0.080 & 0.070 & - & 0.957 & 0.909 & 0.484 & 0.841 & 0.388 & 0.838 \\
C4 & 1.534 & 1.549 & 1.494 & 1.387 & 1.544 & 1.517 & 1.544 & - & 0.344 & 0.538 & 0.884 & 0.574 & 0.859 \\
C5 & 1.363 & 1.384 & 1.346 & 1.236 & 1.379 & 1.330 & 1.380 & 0.072 & - & 0.523 & 0.866 & 0.561 & 0.800 \\
G1 & 1.091 & 1.141 & 1.361 & 1.405 & 1.134 & 1.134 & 1.143 & 1.590 & 1.481 & - & 0.474 & 0.304 & 0.539 \\
G2 & 1.233 & 1.280 & 1.312 & 1.279 & 1.286 & 1.255 & 1.273 & 1.731 & 1.716 & 1.284 & - & 0.506 & 0.828 \\
I1a & 0.391 & 0.415 & 0.482 & 0.509 & 0.399 & 0.400 & 0.442 & 1.149 & 1.152 & 1.014 & 1.181 & - & 0.447 \\
I1b & 0.873 & 0.888 & 0.880 & 0.776 & 0.883 & 0.889 & 0.882 & 0.951 & 0.860 & 1.703 & 1.345 & 0.794 & - \\
\hline
\end{tabular}

Dashes represent zero values; Nei-distances are on the left side; $\mathrm{F}_{\mathrm{ST}}$ are on the right side.

Table 6. Percentage of variation explained by the first three axes using PCoA on microsatellite markers in Figure 4. 


\begin{tabular}{lllll}
\hline Figure & Axis & 1 & 2 & 3 \\
\hline A & $\%$ & 38.57 & 19.76 & 9.22 \\
& Cum \% & 38.57 & 58.33 & 67.55 \\
B & $\%$ & 40.32 & 13.81 & 9.53 \\
& Cum $\%$ & 40.32 & 54.13 & 63.66 \\
\hline
\end{tabular}

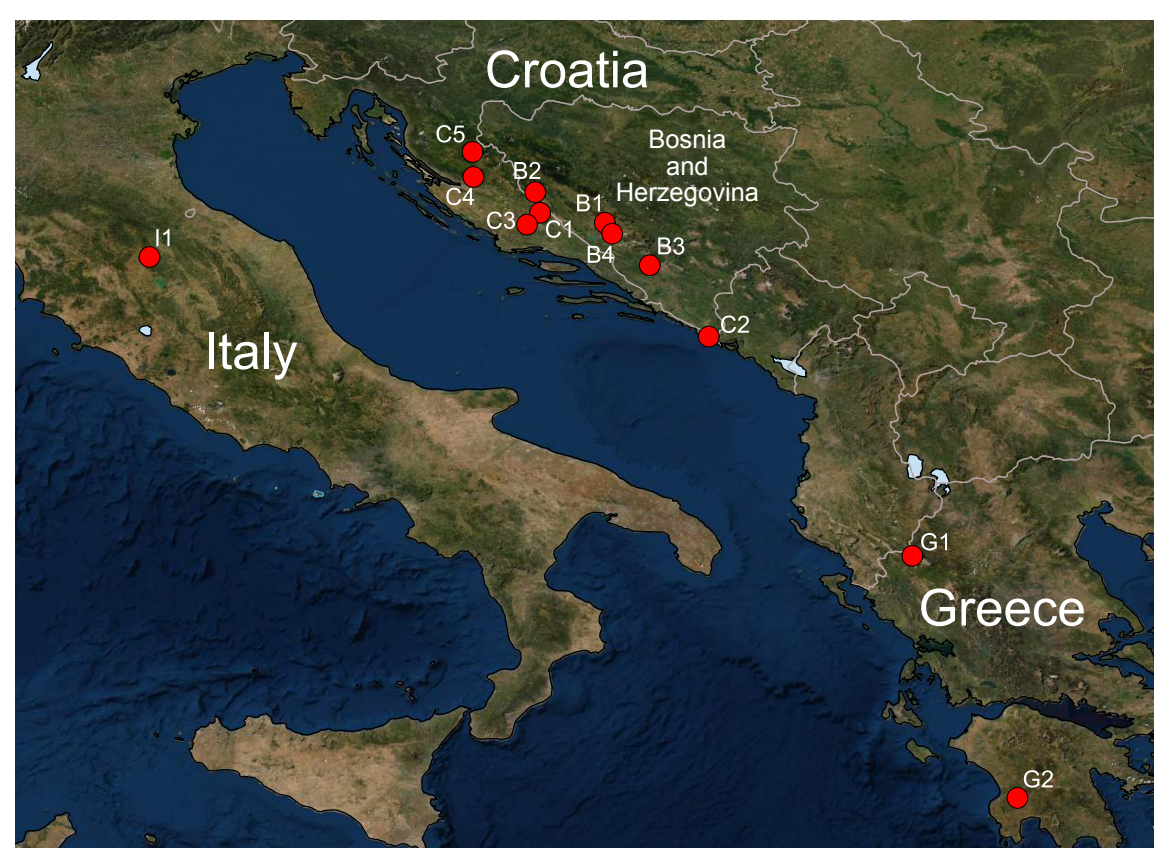



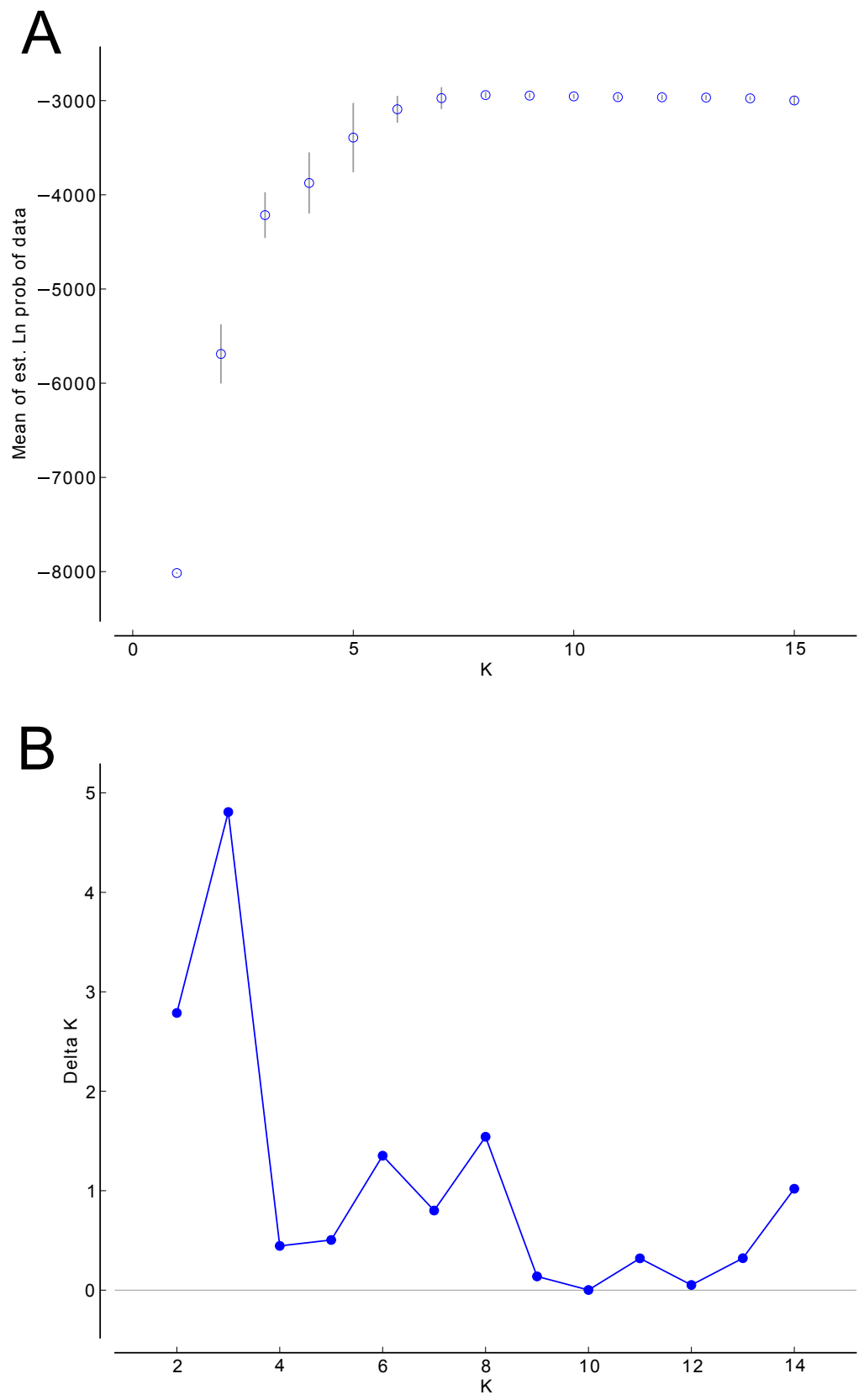


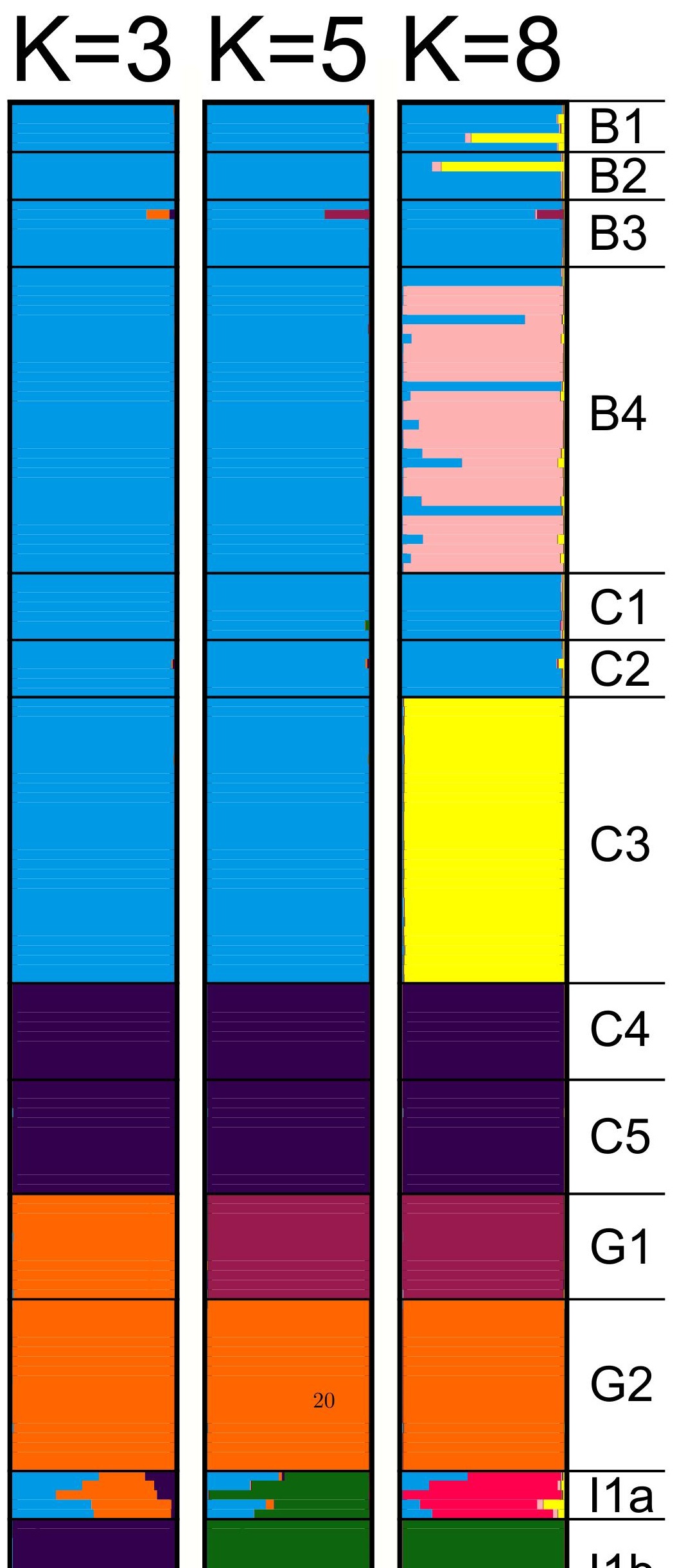


Principal Coordinates (PCoA)
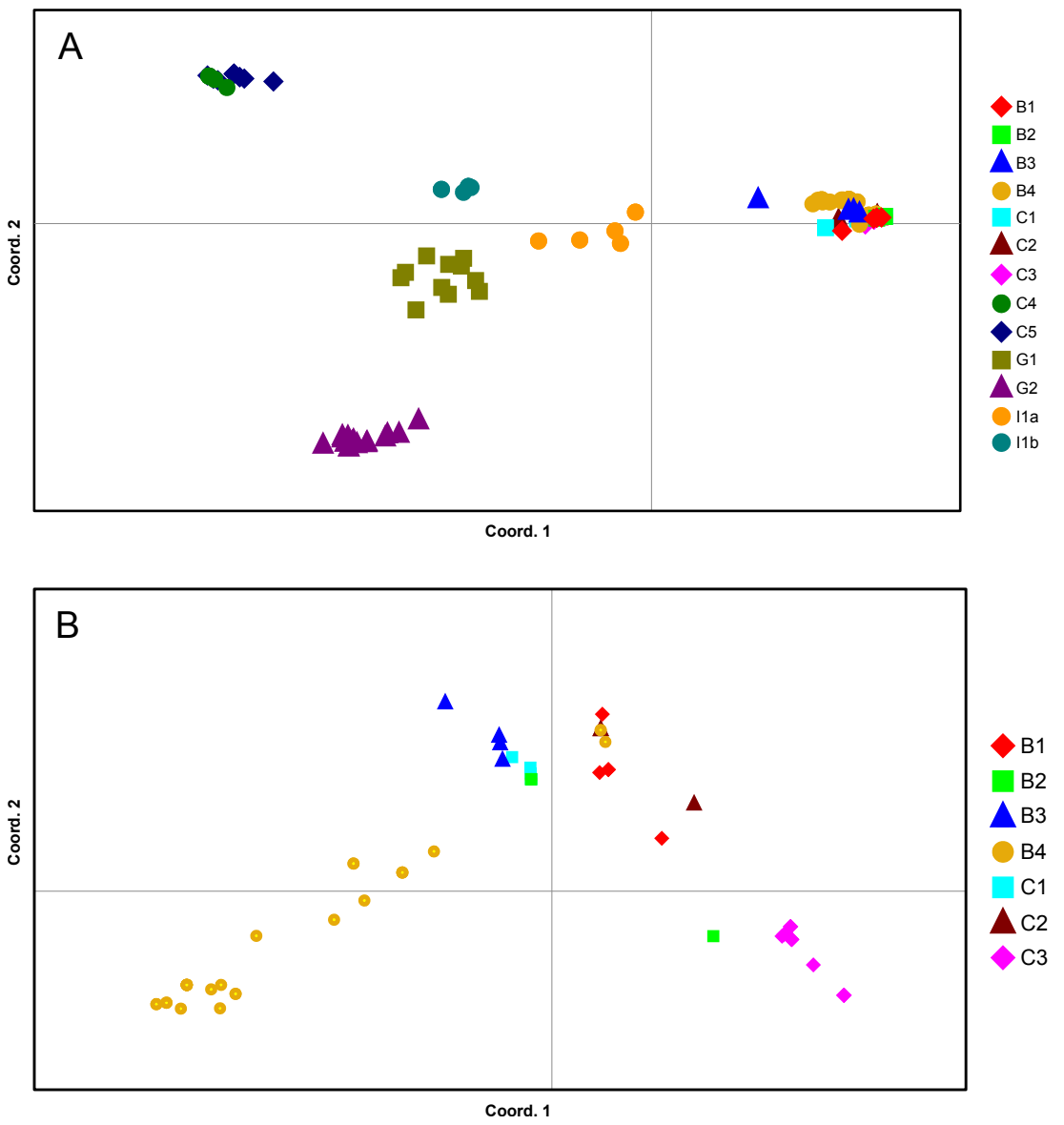